\title{
Diagnóstico del sistema de siembra en invernadero: Casa de malla- laterales abatibles del tomate chonto (Lycopersicon esculentum Mill) en el municipio de Restrepo - Valle del Cauca.
}

\section{Sowing system diagnostic in a greenhouse: nethouse and folding sides of the chonto tomato (Solanum lycopersicon) in the municipality of Restrepo - Valle del Cauca.}

\author{
Alexander Laverde Orozco \\ Agrónomo - Universidad Nacional Abierta y a Distancia \\ alexanderlaverde@sodexo.com \\ María del Pilar Romero Lozada \\ M.Sc. Ciencias Agrarias - Docente Escuela de Ciencias Agrícolas, \\ Pecuarias y del Medio Ambiente \\ mariap.romero@unad.edu.co
}

\section{RESUMEN.}

El tomate de mesa (Lycopersicon esculentum Mill) es una de las hortalizas más sobresalientes en la canasta familiar por su utilización en culinaria. Por la alta incidencia de plagas y enfermedades presenta alta residualidad de agroquímicos en el fruto (Avila et al., 2017). El presente estudio tuvo como objetivo analizar diferentes alternativas de producción con las cuales se procura obtener producciones sanas y rentables soportadas en menores costo de producción. El sistema se fundamenta en el uso de invernaderos que ofrecen estructuras que condicionan factores externos como el clima y aislamiento de insectos plaga y de algunos agentes patógenos, trayendo consigo menor uso de agroquímicos y mejor calidad de fruto. El estudio caracterizó dos modelos de producción del tomate chonto tipo invernadero (casa de malla y cubiertas plásticas con laterales abatibles).

Palabras Clave: Tomate; invernadero; agroquímicos; calidad del fruto; casa de malla; insectos plaga.

\section{ABSTRACT.}

The common tomato (Solanum esculentum) is one of the most outstanding vegetables in the family basket for its use in culinary. Due to the high incidence of pests and diseases, it has high agrochemical residues in the fruit (Avila et al., 2017). The objective of this study was 
to analyze different production alternatives that seek to obtain healthy and profitable productions supported by lower manufacture costs. The system is based on the use of greenhouses that offer structures that condition external factors such as the weather and isolation of pests' insects and some pathogens, resulting in less use of agrochemicals and better fruit quality. The study characterized two models of production of the chonto tomato greenhouse kind (mesh house and plastic covers with folding sides).

Keywords: Tomato; greenhouse; agrochemicals; fruit quality; mesh house; pest insects

\section{INTRODUCCIÓN.}

El tomate de mesa Lycopersicon esculentum es una planta que pertenece a la familia de las solanáceas. El origen de esta hortaliza se localiza en la región Andina, extendiéndose desde Chile hasta Colombia. (Insumos, DANE,2014)

Según la (FAO), en el último decenio en el mundo se cultivaron en promedio 3.37 millones de $\mathrm{Ha}$ de tomate, con un rendimiento de $26.7 \mathrm{t}$. ha-1. (Solis et al., 2006). En Colombia, para el 2016 se encontraron 8450 hectáreas en tomate y una producción de 382.809 toneladas. (DANE-ENA, 2016).

El cultivo del tomate en condiciones invernadero es un tipo de sistema de producción un tanto nueva, en los últimos años ha presentado aumento en área, productividad, rentabilidad y calidad del producto. El rendimiento promedio obtenido con este sistema está entre 5 y $6 \mathrm{~kg} / \mathrm{a}$ diferencia al de campo abierto que esta entre 1,5 y $2 \mathrm{~kg} /$ planta. (Jaramillo, et al., 2007).

La producción de tomate en condiciones de invernadero se basa instalaciones cerradas, cubierta por materiales transparentes 0 semitransparentes estos sistemas que controlan factores climáticos, disminución o aumento ventilación, disminución de la temperatura, aumento de la humedad relativa, evitar riesgos por bajas temperaturas (heladas). Estas características de este sistema de producción reducen la incidencia de plagas y enfermedades, aumenta la producción y rendimiento. Los agricultores del municipio Restrepo - Valle, instalaron varios invernaderos en las diferentes veredas del municipio de Restrepo en respuesta a la alta incidencia de plagas y enfermedades, uso indiscriminado de agroquímicos, al deterioro en la calidad de los frutos y disminución del precio de la hortaliza. En la instalación de estos sistemas de cultivo se poco se tiene en cuenta la asesoría técnica de ahí 
que las instalaciones no se realicen con los materiales adecuados, el diseño apropiado con resultados poco favorables que hace que se desista de este sistema de producción.

De acuerdo a lo anterior se procedió a realizar un análisis de las estructuras existentes en el municipio de Restrepo (V), esto permitió establecer las diferencias, ventajas y limitantes entre los modelos productivos y ser punto de referencia la toma de decisiones en futuros productores de la zona y hacer los respectivos ajustes en los sistemas establecidos.

\section{MARCO TEÓRICO}

Para la producción de cultivos hay opciones de producción, utilizando estructuras protegidas las cuales presentan adaptaciones particulares y de control, teniendo en cuenta el factor ambiental a controlar. Por tratarse de agricultura protegida da lugar a definición, es toda estructura cerrada, cubierta por materiales transparentes 0 semitransparentes que permite dar condiciones especiales de microclima para los cultivos a todo tiempo y bajo condiciones óptimas, con este sistema se realiza el control de las condiciones edafoclimáticas del suelo, temperatura, radiación solar, viento, humedad, entre otros, lo que modifica el ambiente natural en el que se desarrollan los cultivos y así de esta manera producir cultivos con buenas cosechas y rendimientos. (Santos, Obregón-Olivas y Salome -Donoso, 2010).

En cuanto a la protección de cultivos hay variedad de instalaciones con particularidades y estructuras con mayor o menor control ambiental. Entre estos tipos de estructuras de protección de cultivos, se distinguen:

Los acolchados o mulching, este tipo de estructura consiste en colocar materiales orgánicos e inorgánicos sobre el suelo lo que proporciona condiciones para la conservación de la humedad, disminución del crecimiento de plantas arvenses las cuales compiten por agua y nutrientes con el cultivo, mejora la estructura del suelo y el desarrollo de raíces, facilita la fertilidad del suelo. El acolchado o mulch permite el aumento de la temperatura y humedad que facilita la mineralización del suelo, también reduce la erosión causada por las lluvias y viento, evita el contacto directo de los frutos con el suelo protegiéndolo del daño, disminución de la incidencia de plagas y enfermedades.

Las cubiertas flotantes son láminas de materiales plásticos (polietileno, polipropileno, poliéster) que se colocan sobre el cultivo. Estas láminas no presentan estructura que las soporte y a medida que el cultivo crece se eleva el material sin causar daño a las plantas. Esta estructura es un 
método de protección simple, de bajo costo y utilización, proporciona un microclima apto para el crecimiento y desarrollo de las plantas, disminuye la incidencia de plagas y enfermedades.

Los microtúneles se caracterizan por ser estructuras pequeñas conformada por una hilera de arcos los cuales pueden ser tubos, mangueras o alambre grueso, sencillas, de fácil instalación, con una altura de 0.5 a $1 \mathrm{~m}$, cubriendo una o más hileras de cultivo. Esta estructura es utilizada en las primeras etapas del cultivo, ofreciendo protección de los factores climáticos, de plagas y enfermedades.

Los macrotúneles o túneles altos son estructuras generalmente construidas con arcos de bambú, tubos de PVC o hierro galvanizado, cubiertos con una sola capa de plástico de tipo invernadero, agrotextil o malla anti-insectos, presenta una altura entre $3.0 \mathrm{~m}$ y $3.5 \mathrm{~m}$. Por sus características se pueden utilizar variedades indeterminadas y el paso de una o más personas en el interior para el cuidado de plantas. Las dimensiones más utilizadas son de $4 \mathrm{~m}$ de ancho por $30 \mathrm{~m}$ de longitud. La ventilación es pasiva y se realiza enrollando mecánica o manualmente los lados del túnel para permitir la circulación del aire. Los macrotúneles se pueden construir como unidades móviles para permitir la rotación de los cultivos. Proporciona una protección completa al cultivo, lo que se refleja en mayores rendimientos.

Los invernaderos se caracterizan por ser construcciones altas, herméticamente cerradas con materiales transparentes, diseñadas para cultivar o proteger parcialmente a las plantas.

El material del techo y de las paredes frontales y laterales puede ser de diversos materiales entre los que se destacan plástico, vidrio, fibra de vidrio, láminas corrugadas de policarbonato, en el cual se realiza aberturas para una ventilación pasiva, los invernaderos se caracterizan por presentar estructuras rígidas y altas en comparación con otras estructuras, esta estructura permite protección contra diversos factores ambientales como lluvia, calor y frío, menor incidencia de plagas y enfermedades. Por lo tanto, el fin del invernadero es suministrar y mantener un ambiente de crecimiento que produzca cultivos de alta calidad y rendimiento.

Las casas de malla son estructuras que permiten el sostén de mallas de sombra, entre las que se destacan las anti-insectos, anti-pájaros, antigranizo, entre otras protecciones hacia el cultivo. La instalación puede ser fija o móvil. En los semilleros de cultivos y viveros forestales es fundamental la implementación. Este tipo de estructura se caracteriza por la reducción de quemaduras solares hacia el cultivo, reducción de evaporación y evapotranspiración que reduce la demanda de riego y 
aplicación de fertilizantes. Estas condiciones favorecen un mejor desarrollo y productividad.

Los países con mayor área de estructuras protegidas en cultivos son China, España y Japón, con 360,000 Ha, $55000 \mathrm{Ha}$ y $52571 \mathrm{Ha}$, con cultivos como pepino, tomate, pimiento dulce, melón y fresas. (Tabla $1)$.

La mayor área de cultivos protegidos en invernaderos y macrotúneles corresponde a Asia con 440000 (Ha), seguido de Mediterráneo (97.000 $\mathrm{Ha})$, África y Medio Este $(17000 \mathrm{Ha})$, Europa $(16,700 \mathrm{Ha})$ y Américas $(15,000 \mathrm{Ha})$ (Tabla 2).

Tabla 1. Área aproximada de estructuras protegidas en la producción de hortalizas en diferentes países del mundo.

\begin{tabular}{|c|c|c|}
\hline País & Área (Ha) & $\begin{array}{l}\text { Cultivos (en orden de } \\
\text { importancia) }\end{array}$ \\
\hline China & 360,000 & $\begin{array}{l}\text { Pepino, tomate, } \\
\text { pimiento dulce }\end{array}$ \\
\hline España & 55,000 & $\begin{array}{l}\text { Melón, tomate, pimiento } \\
\text { dulce }\end{array}$ \\
\hline Japón & 52571 & Tomate, Melón, fresas \\
\hline Italia & 26000 & $\begin{array}{l}\text { Tomate, Calabaza, } \\
\text { Pimiento dulce }\end{array}$ \\
\hline Corea & 21061 & $\begin{array}{l}\text { Pepino, repollo chino, } \\
\text { tomate }\end{array}$ \\
\hline Noreste de África & 11400 & $\begin{array}{l}\text { Tomate, pimiento dulce, } \\
\text { pepino }\end{array}$ \\
\hline Turquía & 10800 & Tomate, pepino, melón \\
\hline Holanda & 10800 & $\begin{array}{l}\text { Tomate, pimiento dulce, } \\
\text { pepino }\end{array}$ \\
\hline Francia & 9100 & Tomate, pepino y fresas \\
\hline Estados Unidos & 5000 & $\begin{array}{l}\text { Tomate, } \\
\text { lechuga }\end{array}$ \\
\hline Grecia & 4620 & $\begin{array}{l}\text { Tomate, } \\
\text { berenjena }\end{array}$ \\
\hline Medio Este & 4300 & $\begin{array}{l}\text { Tomate, } \\
\text { pimiento dulce. }\end{array}$ \\
\hline Alemania & 3300 & $\begin{array}{l}\text { Tomate, } \\
\text { lechuga }\end{array}$ \\
\hline México & 3200 & Tomate, pimiento dulce \\
\hline Bélgica & 2250 & $\begin{array}{l}\text { Tomate, lechuga, } \\
\text { hierbas, pepino }\end{array}$ \\
\hline Península Arábica & 1930 & Pepino, tomate \\
\hline Noreste de África & 1700 & Pepino, pimiento dulce, \\
\hline
\end{tabular}




\begin{tabular}{|l|l|l|}
\hline & & tomate \\
\hline Reino Unido & 1600 & $\begin{array}{l}\text { Tomate, pepino, } \\
\text { lechuga }\end{array}$ \\
\hline Canadá & 1470 & $\begin{array}{l}\text { Tomate, pepino y } \\
\text { pimiento dulce }\end{array}$ \\
\hline
\end{tabular}

Fuente. (Santos, et al, 2010)

Tabla 2. Área estimada de cultivos protegidos (invernaderos y macrotúneles) por regiones a nivel mundial.

\begin{tabular}{|l|l|}
\hline Región & Área (Ha) \\
\hline Asia & 440.000 \\
\hline Mediterráneo & 97.000 \\
\hline África y Medio Este & 17.000 \\
\hline Europa & 16.700 \\
\hline Américas & 15.600 \\
\hline Total & 586.300 \\
\hline
\end{tabular}

En esta tabla se excluye a los países del mediterráneo. Fuente (Santos et al, 2010).

El tomate de mesa Lycopersicon esculentum es una planta que pertenece a la familia de las solanáceas. El origen de esta hortaliza se localiza en la región Andina, extendiéndose desde Chile hasta Colombia. (Insumos, DANE,2014).

Según la (FAO), en el último decenio en el mundo se cultivaron en promedio 3.37 millones de $\mathrm{Ha}$ de tomate, con un rendimiento de 26.7 t.ha-1. (Solis et al., 2006). En Colombia, para el 2016 se encontraron 8450 hectáreas en tomate y una producción de 382.809 toneladas. (DANE-ENA, 2016).

El cultivo del tomate en condiciones invernadero es un tipo de sistema de producción un tanto nueva, en los últimos años ha presentado aumento en área, productividad, rentabilidad y calidad del producto. El rendimiento promedio obtenido con este sistema está entre 5 y $6 \mathrm{~kg} / \mathrm{a}$ diferencia al de campo abierto que esta entre 1,5 y $2 \mathrm{~kg} /$ planta. (Jaramillo, et al., 2007).

La producción de tomate en condiciones de invernadero se basa instalaciones cerradas, cubierta por materiales transparentes o semitransparentes estos sistemas que controlan factores climáticos, disminución o aumento ventilación, disminución de la temperatura, aumento de la humedad relativa, evitar riesgos por bajas temperaturas (heladas). Estas características de este sistema de producción reducen la incidencia de plagas y enfermedades, aumenta la producción y rendimiento. Los agricultores del municipio Restrepo - Valle, instalaron 
varios invernaderos en las diferentes veredas del municipio de Restrepo en respuesta a la alta incidencia de plagas y enfermedades, uso indiscriminado de agroquímicos, al deterioro en la calidad de los frutos y disminución del precio de la hortaliza. En la instalación de estos sistemas de cultivo se poco se tiene en cuenta la asesoría técnica de ahí que las instalaciones no se realicen con los materiales adecuados, el diseño apropiado con resultados poco favorables que hace que se desista de este sistema de producción.

De acuerdo a lo anterior se procedió a realizar un análisis de las estructuras existentes en el municipio de Restrepo (V), esto permitió establecer las diferencias, ventajas y limitantes entre los modelos productivos y ser punto de referencia la toma de decisiones en futuros productores de la zona y hacer los respectivos ajustes en los sistemas establecidos.

\section{Materiales y métodos}

La investigación se realizó en el municipio de Restrepo - Valle del Cauca. Se realizaron visitas de campo en las 28 veredas que conforman el municipio y en la cabecera municipal, para la identificación de los agricultores con invernadero con cultivo de tomate chonto en el cual se identificaron el tipo de diseño de invernadero: Casa de malla o estructura tipo cubierta plástica con laterales abatibles, tipo de materiales. Para el análisis de los cultivos se realizaron encuestas semiestructuradas dirigidas a los agricultores sobre los procesos de producción del cultivo teniendo en cuenta la variedad o hibrido, rendimiento.

La siembra de tomate en los invernaderos consistió en el tipo chonto, estos presentan forma redonda a ovalada, levemente elongados a oblongos, con dos a cuatro lóculos, con un peso promedio de 70 a 220 gramos. El consumo generalmente es en fresco, se utilizan para la preparación de guisos o pastas. (Jaramillo et al., 2007).

\section{Resultados}

De las veredas 28 veredas visitadas se encontraron estructuras, en la vereda El Aguacate, localizada al sur del municipio, con una altura de $1398 \mathrm{msnm}$ y una temperatura de $27^{\circ} \mathrm{C}$; en esta vereda se identificaron 3 estructuras tipo casa de malla con un área de $1730 \mathrm{~m}^{2}$. En la vereda San Pablo al sur del municipio a $1400 \mathrm{msnm}$ y una temperatura de $25^{\circ} \mathrm{C}$ se encontró una estructura tipo casa de malla con un área de $2000 \mathrm{~m}^{2}$. En la vereda el Silencio con una altura de $1664 \mathrm{msnm}$ y una temperatura 
de $26^{\circ} \mathrm{C}$, se encontraron 8 cubiertas en plástico con cubiertas abatibles, con un área de $8850 \mathrm{~m} 2$.

\section{Descripción de las estructuras}

Las estructuras encontradas en general son de tipo simple no climatizadas, las estructuras son de cubierta plástica con laterales abatibles, está constituida por guadua de forma rectangular, el techo está cubierto en plástico transparente calibre 7 con filtro UV a 2 aguas, con una ventana cenital en la parte superior, la altura promedio de suelo a techo es de 2.50 a 3.00 metros en los costados y de entre 5.00 a 6.00 metros en el centro, los costados cuentan con polisombra la cual se puede bajar o subir según criterio del agricultor, este sistema de cortinas permite regular la temperatura del interior evitando los microclimas que favorecen el desarrollo de patógenos, en tanto la cubierta plástica evita el ingreso del agua lluvia reduciendo la humedad en el follaje siendo el sistema muy eficiente en el control climático de la plantación. Las cubiertas plásticas con laterales abatibles protegen a los cultivos de las heladas que son frecuentes en la vereda el silencio. ( Laverde, 2017). Una desventaja en este tipo de estructura se presenta cuando las cortinas laterales están arriba, permitiendo en el día la entrada de insectos plaga que pueden ocasionar altas infestaciones siendo necesario la aplicación de insecticidas (Insumos- DANE, 2014).

\section{Estructura tipo casa de Malla.}

Este tipo de estructuras tiene postes de guadua, su diseño generalmente es rectangular, esta revestida en su totalidad con malla sintética de alta resistencia tipo anti trips que evita el ingreso de pequeños insectos, la estructura no posee cubierta plástica lo cual deja expuesta al cultivo de las lluvias. En este tipo de estructura la circulación del aire al interior es limitada generando microclimas con alta humedad relativa propiciando ambientes ideales para la diseminación de 
patógenos. Para evitar la proliferación de patógenos en el cultivo se debe realizar con regularidad la eliminación de rebrotes y aún más en las épocas de Iluvia. El agricultor para manejar esta situación debe realizar de manera permanente eliminación de rebrotes para permitir la circulación del aire y de las aspersiones. La principal ventaja de este tipo de invernadero es el aislamiento, pues no permite la entrada de insectos y por ende la disminución de agroquímicos.

El comportamiento de los híbridos (cumanday y Karina) de tomate utilizados en los invernaderos: En las estructuras laterables abatibles y casa de malla; alcanzaron los mínimos fijados por las casas comerciales: de 6 a 7 racimos por planta lo que equivale 5.1 kilogramos y el peso de los frutos para el hibrido para Cumandy es de $180 \mathrm{~g}$ y $190 \mathrm{~g}$ para Karina. (Laverde, 2017).

\section{CONCLUSIONES.}

Funcionalmente los sistemas de Casas de Malla logran una diminución en el uso de los agroquímicos en comparación con las Cubiertas Plásticas con Laterales Abatibles.

Los dos tipos de invernadero tienen como base estructural la guadua (Angustifolia Kunth).

Los productores consideran estos modelos como una alternativa para continuar la actividad agrícola ejerciendo control sobre los factores que afectan la plantación.

\section{BIBLIOGRAFÍA.}

Avila-Orozco, F. D., León-Gallón, L. M., Pinzón-Fandiño, M. I., LondoñoOrozco, A., \& Gutiérrez-Cifuentes, J. A. (2017). Residualidad de fitosanitarios en tomate y uchuva cultivados en Quindío (Colombia). Corpoica Ciencia y Tecnología Agropecuaria, 18(3), 571-582.a. Recuperado de 
http://www.scielo.org.co/pdf/ccta/v18n3/0122-8706-ccta-18-0300571.pdf

Departamento Administrativo Nacional de estadísticas (2017). Encuesta Nacional Agropecuaria ENA 2016. Recuperado de https://www.dane.gov.co/files/investigaciones/agropecuario/enda/ena/2 016/boletin_ena_2016.pdf

Insumos, D. A. N. E. factores asociados a la producción agropecuaria. Bogotá, diciembre 2014. Boletín mensual, (30).

Jaramillo, J., Rodríguez, V., Guzmán, M., Zapata, M., \& Rengifo, T. (2007). Manual técnico: buenas prácticas agrícolas (BPA) en la producción de tomate bajo condiciones protegidas (en línea). Recuperado de http://www.fao.org.co/manualtomate.pdf

Laverde, A. (2017). Caracterización de sistemas productivos de tomate chonto (Lycopersicon esculentun Mill) bajo condiciones tecnificadas en el municipio de Restrepo- Valle del Cauca. Universidad Nacional Abierta y a Distancia, Palmira. Colombia.

Sakata. (05 de 2017). http://www.sakata.com.mx/. Recuperado de http://www.sakata.com.br/cas/productos/hortalizas/solanaceas/tomate

Santos, B., Obregón-Olivas, H., \& Salamé-Donoso, T. (2010). Producción de hortalizas en ambientes protegidos: estructuras para la agricultura protegida. Wimauma, Florida, EE. UU.: University of Florida. Recuperado de https://horticulture.ucdavis.edu/sites/g/files/dgvnsk1816/files/extension _material_files/Santos_academic_paper_estructuras_para_la_agricultur a_protegida.pdf

Solís, A., \& Martínez, R., \& Moya, C., \& Dominí, M., \& López, V., \& Milan, E., \& Amat, I. (2006). Comportamiento de variedades de tomate (Lycopersicon esculentum, Mill) en dos períodos de siembra en la localidad de Velasco, Provincia Holguín. Cultivos Tropicales, 27 (1), 5154. Recuperado de http://www.redalyc.org/articulo.oa?id=193215885010 